\title{
TRASTORNOS DE PERSONALIDAD EN PACIENTES AQUEJADOS DE ANOREXIA Y BULIMIA NERVIOSA: UN ESTUDIO PILOTO
}

\author{
ENRIQUE ECHEBURÚA ${ }^{1}$, IZASKUN MARAÑÓN ${ }^{1}$ y JORGE GRIJALVO ${ }^{2}$ \\ ${ }^{1}$ Facultad de Psicología, Universidad del País Vasco \\ ${ }^{2}$ Servicio Vasco de Salud (Osakidetza)
}

(Aceptado en febrero de 2002)

\begin{abstract}
En este artículo se comparó el perfil de trastornos de personalidad en 25 pacientes con un trastorno de la conducta alimentaria (5 con anorexia restrictiva, 7 con anorexia purgativa, 5 con bulimia purgativa y 8 con un trastorno de la conducta alimentaria inespecífico). El $72 \%$ de las pacientes presentaba, al menos, un trastorno de personalidad, pero no había diferencias de frecuencia entre los cuatro grupos. Más tarde, las 25 pacientes se dividieron en función de la presencia o ausencia de conductas purgativas. Las pacientes purgativas presentaron trastornos de personalidad, de forma estadísticamente significativa, con mayor frecuencia y, además, en mayor número. Se comentan las líneas futuras de investigación en relación con este tema.
\end{abstract}

Palabras clave: Trastornos de la conducta alimentaria, trastornos de personalidad, comorbilidad.

Personality disorders in patients affected by anorexia and bulimia nervosa: A pilot-study

In this paper was compared the personality profile in 25 outpatients with eating disorders (5 with restricting anorexia nervosa, 7 with purging anorexia nervosa, 5 with purging bulimia nervosa and 8 with eating disorder not otherwise specified). Although $72 \%$ of the females showed at least one personality disorder, there were not differences among groups. Later on, all the patients were divided in two groups, according to the presence or absence of purgative behaviors. Patients with purgative behaviors showed more frequently and had a higher number of personality disorders than patients without purging behaviors. Finally implications for further research in this area are commented upon.

Key words: Eating disorders, personality disorders, comorbidity.

\section{INTRODUCCIÓN}

La comorbilidad de las alteraciones de la conducta alimentaria con los trastornos de personalidad ha comenzado a estudiarse recientemente, sobre todo a partir de la inclusión de estos últimos en el eje II del DSM y del desarrollo específico

Correspondencia: Enrique Echeburúa, Facultad de Psicología, Universidad del País Vasco, Av. de Tolosa 70. 20018 Sen Sebastián. Correo-e: ptpodece@ss.ehu.es Agradecimientos. Este estudio, llevado a cabo en el marco de un convenio de investigación entre la Universidad del Pais Vasco y Osakidetza (Servicio Vasco de Salud), ha sido financiado por la Universidad del País Vasco (código del proyecto: UPV 00006.231-H-13931/2001). La segunda autora ha contado con una beca de investigación predoctoral del Gobierno Vasco (código $\mathrm{n}^{2}$ BFI00.113). para su valoración de entrevistas estructuradas como el IPDE (Loranger, 1995) o el SCID-II (Spitzer et al., 1987), así como de pruebas de autoinforme, como el MCM-II (Millon, 1997) (Matsunaga et al., 1998).

Los trastornos de la conducta alimentaria se caracterizan por graves alteraciones en las pautas de alimentación y se han convertido en un problema en ascenso, sobre todo entre las chicas adolescentes y jovenes. En concreto, la principal característica de la anorexia nerviosa es el rechazo a mantener el peso corporal en los valores mínimos normales (IMC > 17,5 , según el criterio de la Organización Mundial de la Salud; IMC = índice de 
masa corporal) y se puede manifestar en forma de dietas drásticas y de ejercicio físico excesivo (anorexia restrictiva) o en forma de atracones y conductas de purga inadecuadas (anorexia compulsiva/purgativa). A su vez, la bulimia se caracteriza por constantes atracones y el uso de métodos compensatorios inapropiados para evitar la ganancia de peso, como los vómitos o el uso inadecuado de laxantes y diuréticos (bulimia purgativa) o, en otros casos, el ayuno y la práctica de ejercicio excesivo (bulimia no purgativa). Por último, hay otras alteraciones de la conducta alimentaria no especificadas, como la sobreingesta compulsiva o ciertos tipos de obesidad mórbida.

Los trastornos de personalidad, por su parte, se corresponden con pautas de conducta inflexibles y no adaptativas, estables temporalmente y que reflejan alteraciones globales de la persona (Echeburúa y Corral, 1999; Sarason y Sarason, 1996; Vázquez et al., 1990). Estos trastornos pueden estar presentes entre el 20 y $\mathbf{4 0} \%$ de los pacientes psiquiátricos ambulatorios.

En general, la comorbilidad de las alteraciones de la conducta alimentaria con los trastornos de personalidad es muy alta: puede oscilar del $20 \%$ al $80 \%$ de los casos (Echeburúa y Marañón, 2001). Esta frecuente comorbilidad entre ambos trastornos hace más difícil la detección temprana del problema, dificulta el tratamiento y ensombrece el pronóstico terapéutico (Díaz et al., 1999).

El objetivo de este estudio-piloto es, por un lado, conocer la comorbilidad de las alteraciones de personalidad con la anorexia y la bulimia nerviosa y, por otro, comparar la comorbilidad de los trastornos de personalidad con los diferentes tipos de trastornos de la conducta alimentaria atendidos en la Unidad de Atención Específica a los Trastornos de la Conducta Alimentaria de Osakidetza (Servicio Vasco de Salud), que tiene una amplia experiencia en este tema (Grijalvo et al., 2000, 2001). Se trata de conocer con mayor precisión la psicopatología asociada a la anorexia y bulimia y de ofrecer más luz para, en último término, diseñar programas terapéuticos más adecuados a las necesidades específicas de estas pacientes.

\section{MÉTODO}

\section{Participantes}

La muestra está compuesta por 25 mujeres jóvenes ( $M=21,36$ años) que cumplen los criterios del DSM-IV (APA, 1994) para anorexia nerviosa restrictiva (ANR) $(n=5)$, anorexia nerviosa purgativa (ANP) $(n=7)$, bulimia nerviosa purgativa (BNP) $(n=5)$ y trastorno de la conducta alimentaria inespecífico (TAI) $(n=8)$. Las pacientes utilizadas en este estudio-piloto son aquellas que llegaron a la Unidad Específica para el Tratamiento de los Trastornos de la Conducta Alimentaria de Osakidetza (Servicio Vasco de Salud), situada en San Sebastián y que es el centro de referencia para la provincia de Guipúzcoa (750.000 habitantes), entre enero y octubre de 2001.

\section{Pruebas de evaluación}

El diagnóstico de los trastornos de la conducta alimentaria se ha hecho mediante una entrevista clínica con arreglo a las categorías y criterios diagnósticos del DSM-IV. La entrevista ha sido llevada a cabo por un psiquiatra con amplia experiencia clínica (el tercer firmante de este estudio).

Respecto a los trastornos de personalidad, el Millon Clinical Multiaxial Inventory-II (MCMI-II; Millon, 1987) es un cuestionario autoaplicado que consta de 175 ítems y tiene como objetivo poder identificar estados clínicos y trastornos 
de personalidad según la clasificación del DSM-III-R. Así, el MCMI-II contiene ocho escalas básicas de personalidad: (1) Esquizoide, (2) Evitativa, (3) Dependiente, (4) Histriónica, (5) Narcisista, (6A) Antisocial, (6B) Agresivo-Sádica, (7) Compulsiva, (8A) Pasivo-Agresiva y (8B) Autodestructiva. Adicionalmente cuenta con tres escalas de personalidad patológica: (S) Esquizotípica, (C) Límite y (P) Paranoide. El MCMI-II incluye también nueve escalas de síntomas, seis de ellas de gravedad moderada (Ansiedad, Histeriforme, Hipomanía, Neurosis depresiva, Abuso de alcohol, Abuso de drogas) y tres de gravedad acentuada (Pensamiento psicótico, Depresión mayor, Trastorno delirante). Sin embargo, en este estudio no se han analizado estas nueve escalas porque aportan una información que no es significativa para el objetivo de esta investigación.

\section{Procedimiento}

Una vez establecido el diagnóstico clínico del trastorno de la conducta alimentaria, todas las pacientes fueron entrevistadas por la segunda autora de este trabajo y completaron el MCMI-II en el marco de la evaluación previa al tratamiento. Con arreglo a los criterios más conservadores de Weltzler (1990), sólo se ha considerado la presencia de un trastorno de personalidad cuando la puntuación en la tasa-base (TB) del MCMI-II es superior a 84. Así, se han analizado los siguientes datos: (1) la frecuencia de los trastornos de personalidad en las pacien- tes estudiadas, en general y también según el tipo específico de trastorno alimentario experimentado; (2) el número medio de trastornos de personalidad en cada uno de los grupos de trastornos de la conducta alimentaria; (3) la frecuencia de los trastornos de personalidad en función de la presencia o no de conductas purgativas; y (4) la presencia de cada trastorno de personalidad en pacientes purgativas y no purgativas. Todas las frecuencias han sido analizadas mediante el test de Chi-cuadrado. Asimismo se ha utilizado un ANOVA de un factor para hacer la comparación de las medias del número de trastornos en cada grupo, con la prueba de Tuckey como análisis post hoc.

\section{RESULTADOS}

El $72 \%$ de las pacientes estudiadas presenta, al menos, un trastorno de personalidad, pero no hay diferencias estadísticamente significativas entre los diferentes grupos (Tabla 1). Sin embargo, más allá de la frecuencia global, sí hay diferencias en el número de trastornos de personalidad sufridos. En concreto, las pacientes con anorexia purgativa presentan como promedio más trastornos de personalidad $(M$ $=2,29)$ que aquellas con anorexia restrictiva $(M=0,40)(F=4,184 ; p<0,05)$ (Tabla 2$)$.

El perfil de trastornos de personalidad en los distintos grupos de la conducta alimentaria no es muy específico ni permite detectar diferencias significativas, pero refleja algunas tendencias. En general, las pacientes con anorexia restrictiva

Tabla 1. Frecuencia de trastornos de personalidad en pacientes con trastornos de la conducta alimentaria

\begin{tabular}{|c|c|c|c|c|c|c|c|c|c|c|}
\hline \multicolumn{2}{|c|}{$\begin{array}{c}\text { ANR } \\
(n=5)\end{array}$} & \multicolumn{2}{|c|}{$\begin{array}{c}\text { ANP } \\
(n=7)\end{array}$} & \multicolumn{2}{|c|}{$\begin{array}{c}\text { BNP } \\
(n=5)\end{array}$} & \multicolumn{2}{|c|}{$\begin{array}{c}\text { TAI } \\
(n=8)\end{array}$} & \multicolumn{2}{|c|}{$\begin{array}{c}\text { Total } \\
(N=25)\end{array}$} & $x^{2}$ \\
\hline Frec. & $\%$ & Frec. & $\%$ & Frec. & $\%$ & Frec. & $\%$ & Frec. & $\%$ & 5,65 \\
\hline 2 & 40 & 7 & 100 & 3 & 60 & 6 & 75 & 18 & 72 & $n s$ \\
\hline
\end{tabular}

Nota $:$ Frec. $=$ frecuencia. ANR = anorexia nerviosa restrictiva, ANP = anorexia nerviosa purgativa, BNP = bulimia nerviosa purgativa, $\mathrm{TAI}=$ trastorno de la conducta alimentaria inespecifico. 
Tabla 2. Número de trastornos de personalidad según los distintos trastornos de la conducta alimentaria

\begin{tabular}{|c|c|c|c|c|c|c|}
\hline $\begin{array}{l}\mathrm{N}^{2} \text { trastornos de } \\
\text { personalidad }\end{array}$ & $\begin{array}{c}\text { ANR } \\
(n=5)\end{array}$ & $\begin{array}{c}\text { ANP } \\
(n=7)\end{array}$ & $\begin{array}{c}\text { BNP } \\
(n=5)\end{array}$ & $\begin{array}{c}\text { TAI } \\
(n=8)\end{array}$ & $\begin{array}{l}\text { TOTAL } \\
(N=25) \\
\end{array}$ & $x^{2}$ \\
\hline 0 & $3(60 \%)$ & & $2(40 \%)$ & $2(25 \%)$ & $7(28 \%)$ & 12,349 \\
\hline 1 & $2(40 \%)$ & $2(28,6 \%)$ & $1(20 \%)$ & $4(50 \%)$ & $9(36 \%)$ & ns \\
\hline 2 & & $2(28,6 \%)$ & $1(20 \%)$ & $2(25 \%)$ & $5(20 \%)$ & \\
\hline 3 & & $2(28,6 \%)$ & $1(20 \%)$ & & $3(12 \%)$ & \\
\hline 4 & & $1(14,3 \%)$ & & & $1(4 \%)$ & \\
\hline \multirow{2}{*}{$\begin{array}{l}\text { Promedio de } \\
\text { trastornos de } \\
\text { personalidad }\end{array}$} & Media & Media & Media & Media & Media & $F$ \\
\hline & 0,40 & 2,29 & 1,20 & 1 & 1,14 & $\begin{array}{c}4,184^{\star} \\
\text { ANP>ANR }\end{array}$ \\
\hline
\end{tabular}

Nota: ANR = anorexia nerviosa restrictiva, ANP = anorexia nerviosa purgativa, BNP = bulimia nerviosa purgativa, $\mathrm{TAI}=$ trastorno de la conducta alimentaria inespecífico.

${ }^{\star} \mathrm{p}<0,05$.

presentan un perfil esquizoide y pasivoagresivo; las pacientes con anorexia purgativa, un patrón esquizoide, con rasgos fóbicos y agresivo-sádicos; las pacientes con bulimia purgativa, un perfil pasivoagresivo y límite; $\mathrm{y}$, por último, las pacientes con un trastorno alimentario inespecífico, un patrón más bien histriónico.

Por otra parte, se ha agrupado a las pacientes de los cuatros grupos de los trastornos de la conducta alimentaria en función de la presencia o no de conduc- tas purgativas y se han analizado los trastornos de personalidad según este criterio. De este modo, las pacientes purgativas presentan trastornos de personalidad, de forma estadísticamente significativa, con mayor frecuencia (Tabla 3) y, además, en mayor número (Tabla 4).

Por último, se presenta el perfil de los trastornos de personalidad con arreglo a estas dos categorías analizadas (pacientes purgativas y pacientes no purgativas). En general, en las pacientes purgativas son más frecuentes los trastornos pasivo-agre-

Tabla 3. Frecuencia de trastornos de personalidad en pacientes purgativas y no purgativas

\begin{tabular}{ccccc}
\hline \multicolumn{2}{c}{$\begin{array}{c}\text { Pacientes purgativas } \\
(n=13)\end{array}$} & \multicolumn{2}{c}{$\begin{array}{c}\text { Pacientes no purgativas } \\
(n=9)\end{array}$} & \multirow{2}{*}{$\chi^{2}$} \\
\hline Frecuencia & $\%$ & Frecuencia & $\%$ & \multirow{3}{*}{$3,956^{\star}$} \\
\hline 11 & $\mathbf{8 4 , 6}$ & 4 & 44,4 & \\
\hline
\end{tabular}

* $\mathrm{p}<0,05$.

Tabla 4. Número de trastornos de personalidad en pacientes purgativas y no purgativas

\begin{tabular}{cccc}
\hline $\begin{array}{c}\text { No trastornos de } \\
\text { Personalidad }\end{array}$ & $\begin{array}{c}\text { Pacientes purgativas } \\
(n=13)\end{array}$ & $\begin{array}{c}\text { Pacientes no purgativas } \\
(\mathbf{n}=\mathbf{9})\end{array}$ & $\chi^{2}$ \\
\hline 0 & $2(15,4 \%)$ & $5(55.6 \%)$ & 5,896 \\
1 & $4(30,8 \%)$ & $3(33,3 \%)$ & $n s$ \\
2 & $3(23,1 \%)$ & $1(11,1 \%)$ & \\
3 & $3(23,1 \%)$ & & $t$ \\
4 & $1(7,7 \%)$ & & $-2,637^{\star}$ \\
\hline Promedio de & Media & Media & \\
trastornos de & 1,77 & 0,56 & \\
personalidad & &
\end{tabular}

$\star \mathrm{p}<0,05$. 
sivo, esquizoide y agresivo-sádico; en las no purgativas, el esquizoide (Tabla 5).

\section{DISCUSIÓN}

Ésta es la primera investigación en que se hace un estudio de los trastornos de personalidad en los diferentes tipos de trastornos de la conducta alimentaria según la tipología establecida por el $D S M-I V$. Los estudios publicados hasta la fecha se limitan a analizar las diferencias de personalidad en categorías más genéricas de trastornos de la conducta alimentaria, como anorexia y bulimia (por ejemplo, Joiner et al., 2000; Matsunaga et al., 2000). Sin embargo, la limitación más importante de esta investigación, que se justifica por su carácter de estudio-piloto, es el pequeño tamaño de la muestra. No obstante, pueden obtenerse ciertas conclusiones que son sugerentes y que aportan una perspectiva nueva a las investigaciones ya establecidas.

La conclusión más relevante es que el $72 \%$ de la muestra total de pacientes con anorexia y bulimia nerviosa (es decir, 2 de cada 3) presentan, al menos, un trastorno de personalidad. Este resultado es comparable a los obtenidos por diferentes autores, como Wonderlich et al.
(1990), Norman et al. (1993) y Grilo et al. (1996). Este hecho supone un reto en la práctica clínica, ya que, como citan Díaz et al. (1999), la presencia de un trastorno de la personalidad junto a una anorexia o una bulimia complica el tratamiento y el pronóstico de este cuadro clínico.

Por lo que se refiere a los diferentes grupos de trastornos de la conducta alimentaria, los resultados estadísticos, sin ser concluyentes, aportan algunas tendencias. En concreto, las pacientes con anorexia purgativa son las que más complicaciones psicopatológicas presentan, por ser en ellas en las que mayor cantidad de trastornos de personalidad se observan. Ello es congruente con una percepción de calidad de vida deficitaria en este subtipo de pacientes (Grijalvo et al., 2000).

Independientemente del tipo del trastorno alimentario implicado, las diferencias más claras están entre las pacientes con conductas purgativas y las pacientes sin ellas. En las primeras aparecen más frecuentemente que en las segundas trastornos de personalidad (especialmente, el pasivo-agresivo, el agresivo-sádico y el esquizoide). Además, lo más habitual en ellas es la presencia de más de un trastorno de personalidad, lo cual ocasiona dificultades adicionales de tratamiento (Wonderlich, 1995).

Tabla 5. Perfil de los trastornos de personalidad en pacientes purgativas y no purgativas

\begin{tabular}{|c|c|c|c|c|c|c|}
\hline \multirow[t]{2}{*}{ Trastornos de personalidad } & \multicolumn{2}{|c|}{ Pacientes purgativas } & \multicolumn{4}{|c|}{ Pacientes no purgativas } \\
\hline & & & & $\%$ & $n$ & 9 \\
\hline Esquizoide & 3 & 23,1 & 2 & & 22 & \\
\hline Fóbica & 2 & 15,4 & 0 & & 0 & \\
\hline Dependiente & 2 & 15,4 & 0 & & 0 & \\
\hline Histriónica & 2 & 15,4 & 1 & & 11 & \\
\hline Narcisista & 0 & 0 & 0 & & 0 & \\
\hline Antisocial & 1 & 7,7 & 0 & & 0 & \\
\hline Agresivo-sádica & 3 & 23,1 & 0 & & 0 & \\
\hline Compulsiva & 1 & 7,7 & 0 & & 0 & \\
\hline Pasivo-agresiva & 6 & 46,2 & 1 & & 11 & \\
\hline Autodestructiva & 1 & 7,7 & 1 & & 11 & \\
\hline Esquizotípica & 0 & 0 & 0 & & 0 & \\
\hline Límite & 2 & 15,4 & 0 & & 0 & \\
\hline Paranoide & 0 & 0 & 0 & & 0 & \\
\hline
\end{tabular}


Una aportación de este estudio, en consonancia con la conclusión anterior, es la significación psicopatológica de las conductas purgativas, al menos desde la perspectiva de los trastornos de personalidad. Ello confiere un valor central al hecho de purgarse y plantea la posibilidad de clasificar a los trastornos de la conducta alimentaria desde esta perspectiva. Se requiere, en todo caso, la existencia de nuevos estudios que confirmen estos hallazgos preliminares.

En adelante, sería conveniente, además de recurrir a muestras más amplias, comparar estos resultados procedentes de la aplicación del MCMI-II con aquellos obtenidos mediante entrevistas clínicas en las que se evalúen los trastornos de personalidad (por ejemplo, el IPDE) (Loranger, 1995). El recurso exclusivo a los autoinformes es muy limitado en el ámbito de los trastornos de personalidad. Es importante también que en los estudios futuros se utilicen grupos de control clínicos, dado que tener un trastorno del eje I aumenta la probabilidad de experimentar un trastorno del eje II. Se trata, en último término, de conocer si los trastornos de personalidad aparecen realmente en las pacientes con trastornos de la conducta alimentaria en mayor medida que en otras poblaciones clínicas.

\section{REFERENCIAS BIBLIOGRÁFICAS}

American Psychiatric Association (APA) (1994). Diagnostic and statistical manual of mental disorders (4th ed.). Washington, D.C.: APA.

Díaz, M., Carrasco, J.L., Prieto, R. y Saiz, J. (1999). El papel de la personalidad en los trastornos de la conducta alimentaria. Actas Españolas de Psiquiatría, 27, 43-50.

Echeburúa, E. y Corral, P. (1999). Avances en el tratamiento cognitivo-conductual de los trastornos de personalidad. Análisis y Modificación de Conducta, 25, 585-614.
Echeburúa, E. y Marañón, I. (2001). Comorbilidad de las alteraciones de la conducta alimentaria con los trastornos de personalidad. Psicología Conductual, 9, 513-525.

Grijalvo, J., Insúa, P. y Iruín, A. (2000). Características y evolución de los pacientes con trastornos de la conducta alimentaria atendidos en un Servicio Público de Salud Mental Extrahospitalaria. Psiquiatría Biológica, 7, 100-108.

Grijalvo, J., Insúa, P. y Iruín, A. (2001). Un modelo grupal para el abordaje de los pacientes con trastornos de la conducta alimentaria atendidos en un Servicio Público de Salud Mental Extrahospitalaria de Osakidetza-Servicio Vasco de Salud. Psiquiatría Biologica, 8, 38-47.

Grilo, C.M., Levy, K.N., Becker, D.F., Edell. W.S. y McGlashan, T.H. (1996). Comorbidity of DSM-III-R Axis I and II disorders among female inpatients with eating disorders. Psychiatric Services, 47, 426-429.

Joiner, E..T., Katz, J. y Heatherton, T.F. (2000). Personality features differentiate late adolescent females and males with chronic bulimic symptoms. International Journal of Eating Disorders, 27, 191-197.

Loranger, A.W. (1995). International Personality Disorder Examination (IPDE). Ginebra: Organización Mundial de la Salud.

Matsunaga, H., Kiriike, N., Nagata, T. y Yamagami, S. (1998). Personality disorders in patients with eating disorders in Japan. International Journal of Eating Disorders, 23, 399-408.

Matsunaga, H., Kaye, W.H., McConaha, C., Plotnicov, K., Pollice, C. y Rao, R. (2000). Personality disorders among subjects recovered from eating disorders. International Journal of Eating Disorders, 27, 353-357.

Millon,T.(1997). Millon Clinical Multiaxial Inventory-II (MCMI-II). Minneapolis: National Computer Systems.

Norman, D., Blais, M.A. y Herzog, D. (1993). Personality characteristics of eating-disordered patients as identified by the Millon clinical multiaxial inventory. Journal of Personality Disorders, 7, 1-9.

Sarason, I.G. y Sarason, B.R. (1996). Psicología anormal ( $7^{a}$ ed.). México: Prentice-Hall. Spitzer, R.L., Williams, J.B. y Gibbon, M. (1987). The Structured Clinical Interview 
for DSM-III-R Personality Disorders. New York: Biometric Research, New York State Psychiatric Institute.

Várquez, C., Ring, J. y Avia., M.D. (1990). Trastornos de la personalidad. En F. Fuentenebro y C. Vázquez (Eds.). Psicología médica, psicopatología y psiquiatría (Vol. 2, pp. 771-806). Madrid: Interamericana. Weltzer, S. (1990). The Millon clinical multiaxial inventory (MCMI): A review. Journal of Personality assessment, 55, 445-464. Wonderlich, S.A. (1995). Personality and eating disorders. En K.D. Brownell y C.G. Fairburn (Eds.). Eating disorders and obsesity: A comprehensive handbook (pp. 171176). New York: Guilford Press.

Wonderlich, S.A., Swift, W.J., Slotnick,H.B. y Goodman, S. (1990). DSM-III-R personality disorders in eating disorders subtypes. International Journal of Eating Disorders, 9, 607-616.

World Health Organization (1992). The ICD10 Classification of Mental and Behavioral Disorders. Geneva: Author. 